\title{
Commentary on "Compatibility of rapid enteral feeding advances and noninvasive ventilation in preterm infants - an observational study"
}

\author{
Catherine Buck ${ }^{1}$ and Sarah Taylor ${ }^{1}$ \\ ${ }^{1}$ Yale School of Medicine
}

January 18,2022

\begin{abstract}
Nutritional support is essential in the critical care of the very preterm infant, however, study of the potential role of nutrition in the multifactorial pathogenesis of bronchopulmonary dysplasia (BPD) is limited. Achievement of full enteral feeding faster has not been shown to be harmful and may be of benefit in extremely preterm infants. This commentary discusses the studied risk and potential benefits of more rapid enteral feeding advancement in extremely preterm infants.
\end{abstract}

Commentary on "Compatibility of rapid enteral feeding advances and noninvasive ventilation in preterm infants - an observational study"

Catherine O. Buck, MD and Sarah N. Taylor, MD, MSCR

Yale School of Medicine, Department of Pediatrics

Corresponding Author:

Sarah N. Taylor

Yale School of Medicine

Department of Pediatrics

333 Cedar Street

PO Box 208064

New Haven, CT 06520

Sarah.n.taylor@yale.edu

Phone: 203-688-2320

Fax: 203-688-5426

Funding:

The authors received no financial support for the research, authorship, and/or publication of this article.

Key words:

extremely preterm infant, enteral feeding, faster feeding advancement

Running title: 
Benefits of faster feeding advancement in preterm infants

\section{Abstract}

Nutritional support is essential in the critical care of the very preterm infant, however, study of the potential role of nutrition in the multifactorial pathogenesis of bronchopulmonary dysplasia (BPD) is limited. Achievement of full enteral feeding faster has not been shown to be harmful and may be of benefit in extremely preterm infants. This commentary discusses the studied risk and potential benefits of more rapid enteral feeding advancement in extremely preterm infants.

Attention to nutritional support is essential in the critical care of the very preterm infant. Both individual nutritional exposures and overall infant growth relate to infant health and neurodevelopmental outcomes. Study of the potential role of nutrition in the multifactorial pathogenesis of bronchopulmonary dysplasia (BPD) is limited. A potential causal pathway is apparent as nutrition is required for both growth and repair of the preterm infant lung which is halted at the saccular stage of development. Observational studies have shown that infants with BPD have suboptimal growth outcomes compared with preterm infants without lung disease, both during the neonatal hospitalization and beyond.(Davidson et al. 1990; Huysman et al. 2003; Vohr et al. 1982; Wang et al. 2010) The directionality of these associations is not clear. The study "Compatibility of rapid enteral feeding advances and noninvasive ventilation in preterm infants an observational study" by Behnke et al focuses on early enteral feeding and its relation to respiratory management in extremely low birthweight infants. The study offers insight into the safety of faster feeding advancement, specifically with the goal to avoid mechanical ventilation by promoting the use of non-invasive ventilation in extremely preterm infants.

\section{Weighing the risks and benefits of faster feeding in preterm infants}

Historically, enteral feeding advancement in the very preterm infant has followed a conservative approach. Some observational studies have reported an association between faster feeding and risk of both necrotizing enterocolitis (NEC) and feeding intolerance. These associations have not been replicated in randomized controlled trials. The SIFT (Speed of Increasing Milk Feeds Trial) study, published in 2019, is the largest randomized multicenter trial of feeding advancement in very preterm infants to date.(Dorling et al. 2019) Among infants born $<32$ weeks gestational age, faster feeding advancement $(30 \mathrm{~mL} / \mathrm{kg} /$ day versus 18 $\mathrm{mL} / \mathrm{kg} /$ day) was not associated with differences in survival without moderate or severe neurodevelopmental impairment at age 24 months. While the SIFT study did not specifically evaluate feeding intolerance, a Cochrane review of 14 randomized trials of fast versus slow feeding advancement found no significant differences in feeding intolerance between groups.(Oddie et al. 2021) In that review, and in the SIFT study, rapid feeding advancement was not associated with NEC, and more importantly, slower feeding advancement did not prevent NEC or neonatal mortality.

Achievement of full enteral feeds more rapidly likely equates to less parenteral nutrition and less central line days.(Fenin et al. 2020) Central line infections are an important risk factor for morbidity and mortality in extremely preterm infants.(Stoll et al. 2002) Studies to date, including the Behnke et al study in this issue, have not shown a significant decrease in late onset sepsis when full enteral feeds are achieved more quickly.(Dorling et al. 2019; Modi et al. 2019; Raban et al. 2016) A recently-updated meta-analysis of 11 trials demonstrated that slow advancement of feeds was associated with an $14 \%$ increased risk of invasive infection (RR 1.14, 95\% CI 0.99 to 1.31) in preterm infants.(Oddie et al. 2021) Late onset sepsis, and more importantly the inflammation associated with this condition, is associated with the development of BPD.(Balany and Bhandari 2015; Jung and Lee 2019) Additional studies are needed to specifically examine the association between earlier enteral feeding achievement and risk of invasive infections in preterm infants. To date, faster feed volume advancement has not be associated with harm and may be associated with benefit.

\section{Preterm infant growth and neonatal lung development}

Preterm infants with BPD have slower growth compared with infants without BPD. This may relate to 
difficulties with nutrient delivery or with medical therapies, such as the use of steroids or diuretics. Nutrient delivery in and of itself is associated with improved pulmonary outcomes.(Wadhawan et al. 2007; Wemhoner et al. 2011) Previous studies have shown that higher energy and caloric intakes in the first postnatal weeks and avoidance of fluid overload are associated with decreased risk of BPD in very preterm infants.(Klevebro et al. 2019; Oh et al. 2005) Additionally, slower weight gain trajectories through 36-weeks corrected gestational age have been associated with an increased incidence of the diagnosis of BPD.(Ehrenkranz et al. 2011; Malikiwi et al. 2019)

Behnke et al found that more rapid feeding advancements $(20-30 \mathrm{~mL} / \mathrm{kg} /$ day versus $10-15 \mathrm{~mL} / \mathrm{kg} /$ day $)$ were associated with improved growth parameters at 36 weeks corrected gestational age, including among infants born small-for-gestational age. These results differ from randomized trials of fast versus slow feeding advancement which have not found more rapid feeding advancement to be associated with improved growth parameters.(Oddie et al. 2021) While infant growth was not the primary outcome of these studies, inconsistencies in findings related to growth may relate to the timing of the feeding intervention.

\section{Early feed initiation for very preterm infants}

The SIFT study began enteral feeding on day 4, after which full enteral feeds were reached over an average of 7 days in the fast group and 11 days in the slow group.(Dorling et al. 2019) Behnke et al began enteral feeds on the first day. Traditionally, neonatal enteral nutrition has been guided by a "fear of feeding". Since enteral nutrition is a risk factor for NEC, the historical clinical approach was to ensure each day that a preterm infant was "safe" to be fed. However, this practice leads to periods with no enteral intake which differs from the fetal experience in which the gut is continuously exposed to amniotic fluid. This lack of amniotic fluid for the preterm infant impacts gut and immunoregulatory development, and this injury is at least partially ameliorated by feeding human milk bioactives. The potential harm of not feeding preterm infants in the first postnatal days suggests neonatal care should consider a move from a "fear of feeding" to a "fear of not feeding", and this evolution is supported by the feed tolerance exhibited in the Behnke et al study and others.(Hamilton et al. 2014) Longer duration of parenteral nutrition has also been associated with altered body composition at term corrected age. (Alja'nini et al. 2021) Regardless of the route of nutrient delivery in the early postnatal days, adequate and balanced early nutritional support is key avoid nutrient deficiencies and optimize neurodevelopmental outcomes.

\section{Nutritional therapies and BPD}

If early and rapid advancement of enteral nutrition relates to a decrease in BPD, what might be the critical nutrient exposure? In a systematic review, Huang et al demonstrated that exclusive and partial human milk diets were associated with lower risk of BPD in preterm infants.(Huang et al. 2019) Patel et al also demonstrated that each $10 \%$ increase in mother's own milk dose was associated with a decreased risk of BPD at 36-week corrected age.(Patel et al. 2017) In the Behnke et al study, in which feedings were initiated on the first postnatal day with standard preterm formula and maternal milk as available, it would be interesting to know the proportion of feeds which were maternal milk.

The specific components of human milk which may protect and/or support lung development include long chain polyunsaturated fatty acids (LC-PUFAs), which promote cell membrane function and surfactant production. Additionally, LC-PUFAs have anti-inflammatory properties, which may have benefits in the repair of neonatal lung injury. Postnatal docosahexaenoic acid (DHA) concentrations were inversely associated with BPD risk in one observational study of infants born $<30$ weeks gestational age.(Martin et al. 2011) Randomized trials of maternal and preterm infant DHA and arachidonic acid supplementation are ongoing. (Wendel et al. 2021) Studies specifically examining the role of PUFAs in reducing risk of lung disease in extremely preterm infants are needed.

Vitamins such as vitamin A, or retinoic acid, and vitamin D also have roles in respiratory outcomes.(Onwuneme et al. 2015; Tyson et al. 1999) Intramuscular injections of vitamin A related to a $7 \%$ reduction in BPD or death in a clinical trial, but similar benefit is not yet shown with enteral supplementation.(Tyson et al. 1999) Further investigation is needed to identify the specific paths by which enteral 
nutrients may mitigate BPD progression.

\section{Feeding tolerance with non-invasive ventilation}

Although the Behnke et al study raises interesting hypotheses as to how achievement of enteral nutrition may relate to $\mathrm{BPD}$, as described by the authors, the most impactful result of this study is the tolerance of enteral nutrition and greater growth trajectory in a very preterm infant population in which $75 \%$ received non-invasive ventilation. As a single-site study, and with large neonatal practice variation in site-specific diagnosis of feeding intolerance, support from further observational studies or clinical trials will strengthen the authors' conclusion. This study calls into question the practice of limiting enteral nutrition advancement in infants receiving non-invasive respiratory support.

\section{Conclusion}

While research continues to work toward determining the best therapies for the prevention of BPD in extremely preterm infants, it remains clear that early and adequate nutritional support in the first postnatal weeks is critical to improve long-term outcomes and prevent harmful neonatal morbidities. As neonatal medicine works toward improving outcomes in the most premature infants, a multifaceted approach to the prevention and treatment of pulmonary outcomes is warranted. In addition to the investigation of the right ventilatory approach to limit injury and oxidative stress to the preterm lung, research strategies which evaluate the role of nutritional treatments aimed to prevent BPD are critical to improving outcomes in extremely preterm infants.

\section{References:}

Alja'nini Z, Merlino-Barr S, Brumfiel A, McNelis K, Viswanathan S, Collin M, Groh-Wargo S. 2021. Effect of parenteral nutrition duration on patterns of growth and body composition in very low-birth-weight premature infants. JPEN J Parenter Enteral Nutr. 45(8):1673-1682.

Balany J, Bhandari V. 2015. Understanding the impact of infection, inflammation, and their persistence in the pathogenesis of bronchopulmonary dysplasia. Front Med (Lausanne). 2:90.

Davidson S, Schrayer A, Wielunsky E, Krikler R, Lilos P, Reisner SH. 1990. Energy intake, growth, and development in ventilated very-low-birth-weight infants with and without bronchopulmonary dysplasia. Am J Dis Child. 144(5):553-559.

Dorling J, Abbott J, Berrington J, Bosiak B, Bowler U, Boyle E, Embleton N, Hewer O, Johnson S, Juszczak $\mathrm{E}$ et al. 2019. Controlled trial of two incremental milk-feeding rates in preterm infants. N Engl J Med. $381(15): 1434-1443$.

Ehrenkranz RA, Das A, Wrage LA, Poindexter BB, Higgins RD, Stoll BJ, Oh W, Eunice Kennedy Shriver National Institute of Child H, Human Development Neonatal Research N. 2011. Early nutrition mediates the influence of severity of illness on extremely lbw infants. Pediatr Res. 69(6):522-529.

Fenin A, Newman JC, Taylor SN. 2020. Very low birth weight infants receive full enteral nutrition within 2 postnatal weeks. J Perinatol. 40(12):1849-1856.

Hamilton E, Massey C, Ross J, Taylor S. 2014. Early enteral feeding in very low birth weight infants. Early Hum Dev. 90(5):227-230.

Huang J, Zhang L, Tang J, Shi J, Qu Y, Xiong T, Mu D. 2019. Human milk as a protective factor for bronchopulmonary dysplasia: A systematic review and meta-analysis. Arch Dis Child Fetal Neonatal Ed. 104(2):F128-F136.

Huysman WA, de Ridder M, de Bruin NC, van Helmond G, Terpstra N, Van Goudoever JB, Sauer PJ. 2003. Growth and body composition in preterm infants with bronchopulmonary dysplasia. Arch Dis Child Fetal Neonatal Ed. 88(1):F46-51. 
Jung E, Lee BS. 2019. Late-onset sepsis as a risk factor for bronchopulmonary dysplasia in extremely low birth weight infants: A nationwide cohort study. Sci Rep. 9(1):15448.

Klevebro S, Westin V, Stoltz Sjostrom E, Norman M, Domellof M, Edstedt Bonamy AK, Hallberg B. 2019. Early energy and protein intakes and associations with growth, bpd, and rop in extremely preterm infants. Clin Nutr. 38(3):1289-1295.

Malikiwi AI, Lee YM, Davies-Tuck M, Wong FY. 2019. Postnatal nutritional deficit is an independent predictor of bronchopulmonary dysplasia among extremely premature infants born at or less than 28 weeks gestation. Early Hum Dev. 131:29-35.

Martin CR, Dasilva DA, Cluette-Brown JE, Dimonda C, Hamill A, Bhutta AQ, Coronel E, Wilschanski M, Stephens AJ, Driscoll DF et al. 2011. Decreased postnatal docosahexaenoic and arachidonic acid blood levels in premature infants are associated with neonatal morbidities. J Pediatr. 159(5):743-749 e741-742.

Modi M, Ramji S, Jain A, Kumar P, Gupta N. 2019. Early aggressive enteral feeding in neonates weighing 750-1250 grams: A randomized controlled trial. Indian Pediatr. 56(4):294-298.

Oddie SJ, Young L, McGuire W. 2021. Slow advancement of enteral feed volumes to prevent necrotising enterocolitis in very low birth weight infants. Cochrane Database Syst Rev. 8:CD001241.

Oh W, Poindexter BB, Perritt R, Lemons JA, Bauer CR, Ehrenkranz RA, Stoll BJ, Poole K, Wright LL, Neonatal Research N. 2005. Association between fluid intake and weight loss during the first ten days of life and risk of bronchopulmonary dysplasia in extremely low birth weight infants. J Pediatr. 147(6):786-790.

Onwuneme C, Carroll A, Doherty D, Bruell H, Segurado R, Kilbane M, Murphy N, McKenna MJ, Molloy EJ. 2015. Inadequate vitamin d levels are associated with culture positive sepsis and poor outcomes in paediatric intensive care. Acta Paediatr. 104(10):e433-438.

Patel AL, Johnson TJ, Robin B, Bigger HR, Buchanan A, Christian E, Nandhan V, Shroff A, Schoeny M, Engstrom JL et al. 2017. Influence of own mother's milk on bronchopulmonary dysplasia and costs. Arch Dis Child Fetal Neonatal Ed. 102(3):F256-F261.

Raban S, Santhakumaran S, Keraan Q, Joolay Y, Uthaya S, Horn A, Modi N, Harrison M. 2016. A randomised controlled trial of high vs low volume initiation and rapid vs slow advancement of milk feeds in infants with birthweights $</=1000 \mathrm{~g}$ in a resource-limited setting. Paediatr Int Child Health. 36(4):288-295.

Stoll BJ, Hansen N, Fanaroff AA, Wright LL, Carlo WA, Ehrenkranz RA, Lemons JA, Donovan EF, Stark AR, Tyson JE et al. 2002. Late-onset sepsis in very low birth weight neonates: The experience of the nichd neonatal research network. Pediatrics. 110(2 Pt 1):285-291.

Tyson JE, Wright LL, Oh W, Kennedy KA, Mele L, Ehrenkranz RA, Stoll BJ, Lemons JA, Stevenson DK, Bauer CR et al. 1999. Vitamin a supplementation for extremely-low-birth-weight infants. National institute of child health and human development neonatal research network. N Engl J Med. 340(25):1962-1968.

Vohr BR, Bell EF, Oh W. 1982. Infants with bronchopulmonary dysplasia. Growth pattern and neurologic and developmental outcome. Am J Dis Child. 136(5):443-447.

Wadhawan R, Oh W, Perritt R, Laptook AR, Poole K, Wright LL, Fanaroff AA, Duara S, Stoll BJ, Goldberg R. 2007. Association between early postnatal weight loss and death or bpd in small and appropriate for gestational age extremely low-birth-weight infants. J Perinatol. 27(6):359-364.

Wang LY, Luo HJ, Hsieh WS, Hsu CH, Hsu HC, Chen PS, Chiu NC, Lee WT, Jeng SF. 2010. Severity of bronchopulmonary dysplasia and increased risk of feeding desaturation and growth delay in very low birth weight preterm infants. Pediatr Pulmonol. 45(2):165-173.

Wemhoner A, Ortner D, Tschirch E, Strasak A, Rudiger M. 2011. Nutrition of preterm infants in relation to bronchopulmonary dysplasia. BMC Pulm Med. 11:7. 
Wendel K, Pfeiffer HCV, Fugelseth DM, Nestaas E, Domellof M, Skalhegg BS, Elgstoen KBP, Rootwelt H, Pettersen RD, Pripp AH et al. 2021. Effects of nutrition therapy on growth, inflammation and metabolism in immature infants: A study protocol of a double-blind randomized controlled trial (imnut). BMC Pediatr. 21(1):19. 